\title{
Abnormal expression of homeobox genes and transthyretin in C9ORF72 expansion carriers
}

\section{OPEN}

NiCole A. Finch, MS

Xue Wang, PhD

Matthew C. Baker, BSc

Michael G. Heckman, MS

Tania F. Gendron, $\mathrm{PhD}$

Kevin F. Bieniek, PhD

Joanne Wuu, ScM

Mariely DeJesus-

Hernandez, BS

Patricia H. Brown, MS

Jeannie Chew, BA

Karen R. Jansen-West, BS

Lillian M. Daughrity, BS

Alexandra M. Nicholson, $\mathrm{PhD}$

Melissa E. Murray, PhD

Keith A. Josephs, MD

Joseph E. Parisi, MD

David S. Knopman, MD

Ronald C. Petersen, MD

Leonard Petrucelli, PhD

Bradley F. Boeve, MD

Neill R. Graff-Radford, MD

Yan W. Asmann, PhD

Dennis W. Dickson, MD

Michael Benatar,

MBChB, DPhil

Robert Bowser, PhD

Kevin B. Boylan, MD

Rosa Rademakers, PhD*

Marka van Blitterswijk,

$\mathrm{MD}, \mathrm{PhD} *$

Correspondence to

Dr. van Blitterswijk:

VanBlitterswijk.Marka@mayo.edu

or

Dr. Rademakers:

Rademakers.Rosa@mayo.edu

Supplemental data at Neurology.org/ng

\section{ABSTRACT}

Objective: We performed a genome-wide brain expression study to reveal the underpinnings of diseases linked to a repeat expansion in chromosome 9 open reading frame 72 (C9ORF72).

Methods: The genome-wide expression profile was investigated in brain tissue obtained from C9ORF72 expansion carriers ( $n=32)$, patients without this expansion $(n=30)$, and controls $(n=20)$. Using quantitative real-time PCR, findings were confirmed in our entire pathologic cohort of expansion carriers $(n=56)$ as well as nonexpansion carriers $(n=31)$ and controls $(n=20)$.

Results: Our findings were most profound in the cerebellum, where we identified 40 differentially expressed genes, when comparing expansion carriers to patients without this expansion, including 22 genes that have a homeobox (e.g., HOX genes) and/or are located within the HOX gene cluster (top hit: homeobox A5 [HOXA5]). In addition to the upregulation of multiple homeobox genes that play a vital role in neuronal development, we noticed an upregulation of transthyretin (TTR), an extracellular protein that is thought to be involved in neuroprotection. Pathway analysis aligned with these findings and revealed enrichment for gene ontology processes involved in (anatomic) development (e.g., organ morphogenesis). Additional analyses uncovered that HOXA5 and TTR levels are associated with C9ORF72 variant 2 levels as well as with intron-containing transcript levels, and thus, disease-related changes in those transcripts may have triggered the upregulation of HOXA5 and TTR.

Conclusions: In conclusion, our identification of genes involved in developmental processes and neuroprotection sheds light on potential compensatory mechanisms influencing the occurrence, presentation, and/or progression of C9ORF72-related diseases. Neurol Genet 2017;3:e161; doi: 10.1212/NXG.0000000000000161

\section{GLOSSARY}

ALS = amyotrophic lateral sclerosis; FTD = frontotemporal dementia; FTLD = frontotemporal lobar degeneration; IQR = interquartile range; MND = motor neuron disease

Amyotrophic lateral sclerosis (ALS) and frontotemporal dementia (FTD) are part of a disease continuum. Although ALS is the most common form of motor neuron disease (MND) and results in progressive muscle weakness, FTD is a frequent cause of dementia and is associated with changes in personality, behavior, and language. A hexanucleotide repeat expansion in chromosome 9 open reading frame 72 (C9ORF72) is a major genetic cause of both diseases. ${ }^{1,2}$ Emerging evidence suggests that C9ORF72-related diseases are characterized by a loss of C9ORF72 expression, ${ }^{1}$ the formation of RNA foci with flawed RNA transcripts, ${ }^{1}$ and the generation of dipeptide repeat proteins aberrantly translated from the repeat expansion, ${ }^{3,4}$ with both RNA foci

*These authors contributed equally to this work as co-last authors.

From the Department of Neuroscience (N.A.F., M.C.B., T.F.G., K.F.B., M.D.-H., P.H.B., J.C., K.R.J.-W., L.M.D., A.M.N., M.E.M., L.P., D. W.D., R.R., M.v.B.), Department of Health Sciences Research (X.W., Y.W.A.), Department of Neurology (N.R.G.-R., K.B.B.), Division of Biomedical Statistics and Informatics (M.G.H.), Mayo Clinic, Jacksonville, FL; Department of Neurology (J.W., M.B.), University of Miami, FL; Department of Neurology (K.A.J., J.E.P., D.S.K., R.C.P., B.F.B.), Mayo Clinic, Rochester, MN; and Divisions of Neurology and Neurobiology (R.B.), Barrow Neurological Institute, St. Joseph's Hospital and Medical Center, Phoenix, AZ.

Funding information and disclosures are provided at the end of the article. Go to Neurology.org/ng for full disclosure forms. The Article Processing Charge was funded by the NIH.

This is an open access article distributed under the terms of the Creative Commons Attribution-NonCommercial-NoDerivatives License 4.0 (CC BY-NC-ND), which permits downloading and sharing the work provided it is properly cited. The work cannot be changed in any way or used commercially without permission from the journal. 
and dipeptide repeat proteins potentially contributing to disease by compromising nucleocytoplasmic transport. ${ }^{5-7}$

In our quest to increase our understanding of C9ORF72-related diseases, we assessed the genome-wide expression profile in brain tissue obtained from the Mayo Clinic Florida Brain Bank $(\mathrm{n}=82)$. Of interest, in C9ORF72 expansion carriers, we discovered an upregulation of genes involved in developmental processes and neuroprotection, particularly in the cerebellum, a region without substantial neuronal loss that demonstrates pathologic hallmarks of C9ORF72-related diseases, ${ }^{3,8}$ and in which abnormalities associate with neuropathologic and clinical phenotypes. ${ }^{9} 10$ Such findings may point toward mechanisms that could compensate for the harmful effects of C9ORF72 repeat expansions.

METHODS Participant selection. From the Mayo Clinic Florida Brain Bank, participants were selected for our genomewide expression study: patients with a pathologic diagnosis of frontotemporal lobar degeneration (FTLD) and/or MND who harbored C9ORF72 repeat expansions $(\mathrm{n}=32)$, patients with FTLD and/or MND without repeat expansions $(\mathrm{n}=30)$, and controls without neurologic diseases $(\mathrm{n}=20$, table 1$)$. To confirm the observed upregulation of homeobox A5 (HOXA5) and transthyretin (TTR), quantitative real-time PCR was performed, when expanding investigations to our entire pathologic cohort of C9ORF72 expansion carriers for whom brain tissue was available $(\mathrm{n}=56)$ as well as FTLD and/or MND patients without an expansion $(\mathrm{n}=31)$ and controls without any neurologic disease $(\mathrm{n}=20) .{ }^{11}$ In this cohort, C9ORF72 transcript levels, the length of the repeat expansion, and dipeptide repeat protein levels had

\begin{tabular}{|llll|}
\hline Table $1 \quad$ Participant characteristics & & \\
$\begin{array}{l}\text { Cohort/variable } \\
\text { Genome-wide expression }\end{array}$ & $\begin{array}{l}\text { C9Plus cohort } \\
(\mathbf{n}=\mathbf{3 2})\end{array}$ & $\begin{array}{l}\text { C9Minus cohort } \\
(\mathbf{n}=\mathbf{3 0})\end{array}$ & $\begin{array}{l}\text { Control cohort } \\
(\mathbf{n}=\mathbf{2 0})\end{array}$ \\
$\begin{array}{l}\text { Sex, male } \\
\text { Age at death, } \mathbf{y}\end{array}$ & $20(63)$ & $12(40)$ & $7(35)$ \\
RIN cerebellum (value) & $63.7(58.4-71.7)$ & $75.0(64.0-81.8)$ & $87.5(81.8-93.0)$ \\
RIN frontal cortex (value) & $9.4(9.2-9.6)$ & $9.2(8.7-9.4)$ & $9.3(8.5-9.4)$ \\
Diagnosis & $9.0(8.5-9.6)$ & $9.1(8.6-9.5)$ & $8.9(8.6-9.2)$ \\
FTLD & $12(38)$ & $10(33)$ & - \\
FTLD/MND & $10(31)$ & $10(33)$ & - \\
MND & $10(31)$ & $10(33)$ & - \\
Other & - & - & - \\
\hline
\end{tabular}

Abbreviations: FTLD = frontotemporal lobar degeneration; IQR = interquartile range; MND = motor neuron disease.

Data are sample median (IQR) or $\mathrm{n}(\%)$. Information was obtained for patients with (C9Plus) and without (C9Minus) expansions in C9ORF72, as well as from controls. This study was performed in the cerebellum and frontal cortex. already been determined. ${ }^{10-12}$ To examine the cerebellar TTR protein, Western blots $(\mathrm{n}=10)$ and immunohistochemistry $(\mathrm{n}=13)$ were performed on a representative subset of samples (table e-1 at Neurology.org/ng). Next, an immunoassay was used to evaluate TTR protein levels in the CSF, studying 2 independent clinical cohorts obtained at either the Mayo Clinic $(n=67)$ or the University of Miami ( $\mathrm{n}=40$, table e-1).

Standard protocol approvals, registrations, and patient consents. All participants agreed to participate in the study, and biological samples were obtained after informed consent with ethical committee approval from the respective institutions.

Methods and statistical analysis. To examine the genomewide expression pattern, Whole-Genome DASL HT assays (Illumina, San Diego, CA) were used, which were processed by the Mayo Clinic Core Facility. Validation was performed with quantitative real-time PCR using TaqMan gene expression assays (Life Technologies, Carlsbad, CA). Western blotting was used to evaluate cerebellar TTR protein levels, complemented with immunohistochemistry to assess the presence of potential TTR protein aggregates. Meso Scale Discovery (MSD, Rockville, MD) electrochemiluminescence detection technology was used to establish a sandwich immunoassay for TTR. Cell culture experiments were then performed in an attempt to clarify underlying mechanisms. In U251 and HepG2 cells, a loss of C9ORF72 expression was mimicked with small interfering RNAs (siRNAs, Dharmacon, Lafayette, CO), and in addition, the effect of fulllength $C 9 O R F 72$ and the repeat expansion itself was examined by transfecting cells with expression vectors. ${ }^{13}$ A detailed description of our methods and statistical analysis is provided in the supplemental data.

RESULTS Upregulation of homeobox genes and $T T R$ in COORF72 expansion carriers. We performed a genome-wide expression study in the cerebellum and frontal cortex to identify genes involved in C9ORF72-related diseases. First, we compared patients with or without a repeat expansion in C9ORF72. Although participants included in those groups are both affected by neurodegenerative diseases, this enabled us to find C9ORF72-specific differences. Second, we compared expansion carriers with controls without neurodegenerative diseases, allowing the detection of more general differences that could, theoretically, be due to the presence of a neurodegenerative disease.

In the cerebellum, when comparing expansion carriers to patients without expansions, we detected 40 differentially expressed genes (table e-2). Generation of a heat map of those genes revealed that expansion carriers generally cluster together (figure 1). Of interest, our list of differentially expressed genes contained 22 genes that have a homeobox (e.g., HOX genes) and/or are located within the $H O X$ gene cluster (table e-2). In addition to the upregulation of multiple homeobox genes (top hit: HOXA5) that play a vital role in neuronal development, ${ }^{14}$ we noticed a cerebellar upregulation of TTR (table e-2), an extracellular protein that is thought to be involved in neuroprotection. ${ }^{15-19} \mathrm{We}$ then compared expansion carriers with controls and 
discovered 1,575 differentially expressed genes in the cerebellum (table e-2). Again, our heat map showed that expansion carriers tend to cluster together (figure 1). Of interest, our new list contained 37 of the 40 (93\%) genes we identified previously (table e-2), including homeobox genes and TTR.

We also performed gene ontology analysis and observed an enrichment for pathways involved in the regulation of (anatomic) development, which was most profound when comparing expansion carriers with disease controls (e.g., organ morphogenesis, pattern specification process, regionalization, and skeletal system development, table e-3), but which was also seen when comparing expansion carriers with controls (table e-3).
In the frontal cortex, a comparison between patients with or without repeat expansions resulted in the detection of 3 differentially expressed genes: HOXA5, C9ORF72, and POU class 4 homeobox 2 (POU4F2; table e-2). We also compared expansion carriers with controls and revealed 679 differentially expressed genes, including C9ORF72 and TTR (table e-2). Again, enrichment was observed for pathways involved in developmental processes (table e-3).

Associations of C9ORF72 transcripts with HOXA5 and TTR transcripts in our overall cohort. In previously published studies, we investigated the levels of known C9ORF72 transcript variants (variant 1 [NM_145005.6], variant 2 [NM_018325.4], and variant 3 [NM_001256054.2])

\section{Figure 1 Expression of homeobox genes and transthyretin}
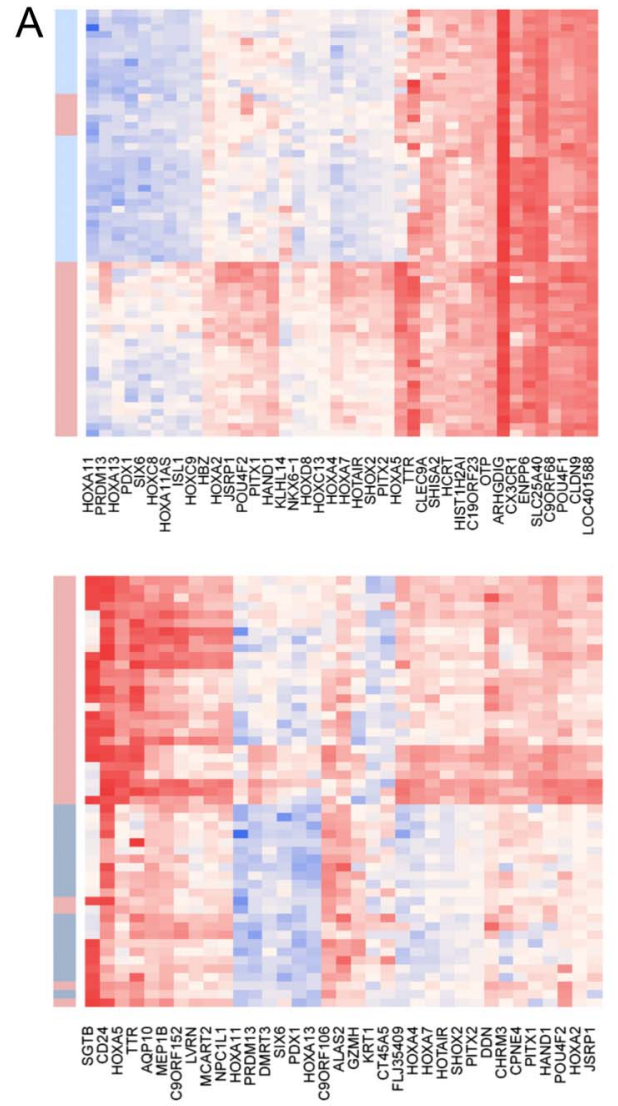

D
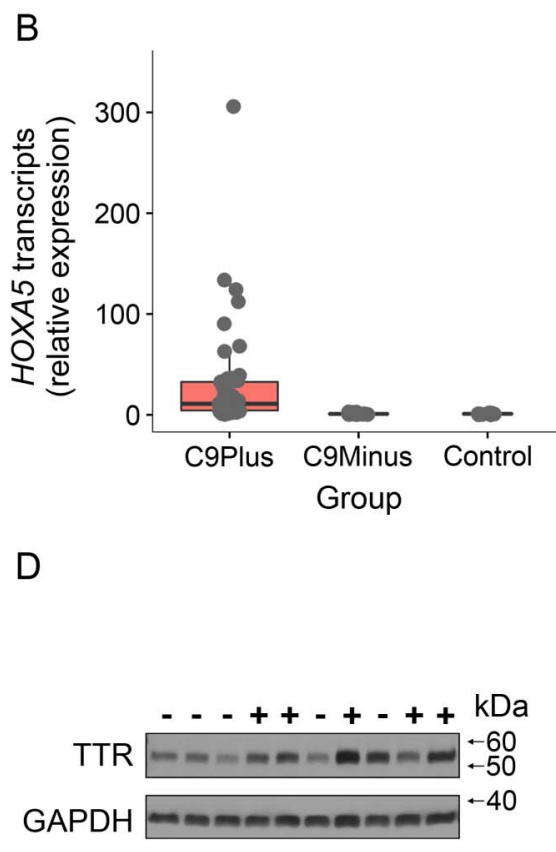

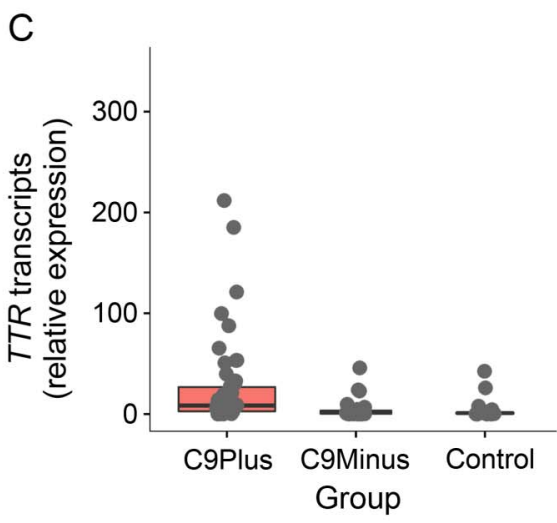

E

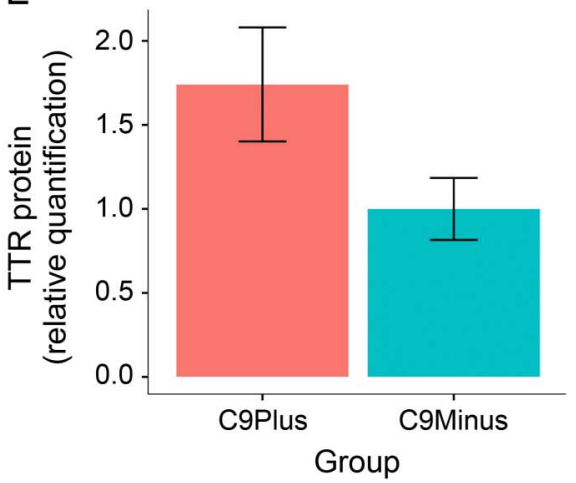

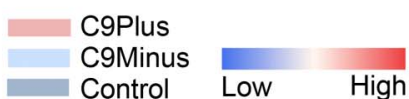

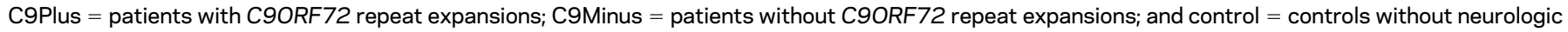

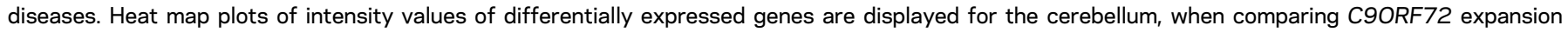

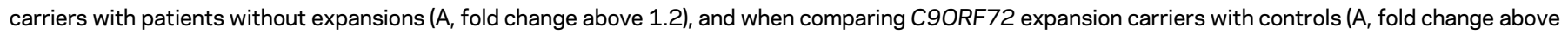

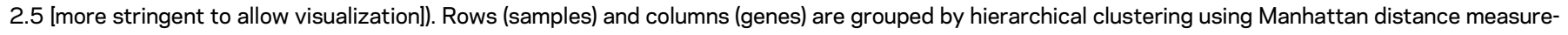

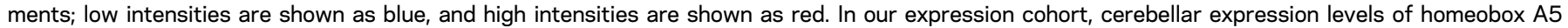

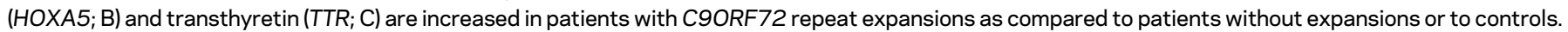

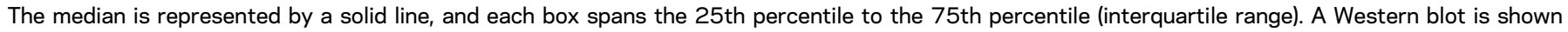

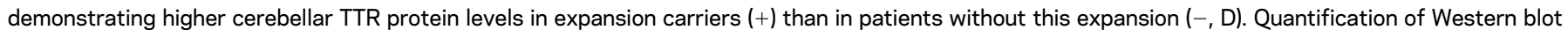

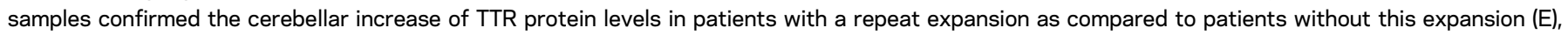

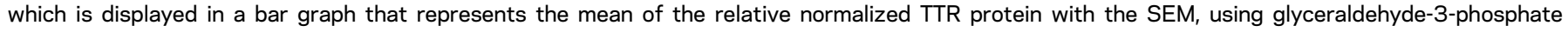
dehydrogenase (GAPDH) as the loading control. 


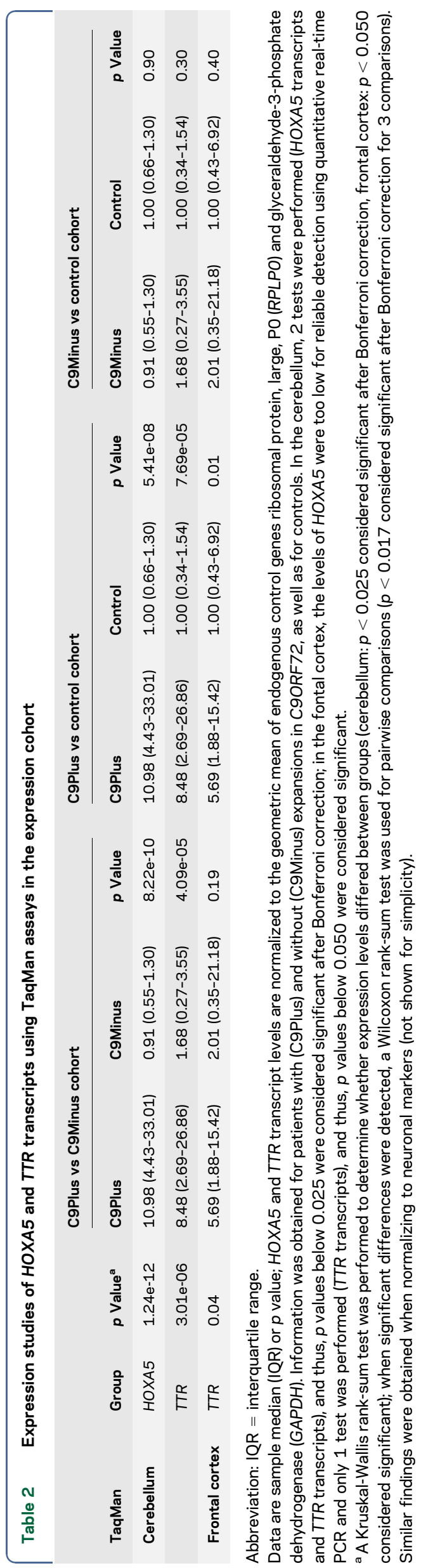

as well as 2 intronic regions ( 1 upstream of the repeat expansion [intron 1a] and 1 downstream of the repeat expansion [intron 1b]). ${ }^{11}$ In this study, we examined the same cohort to validate our findings related to HOXA5 and $T T R$, which demonstrated that their cerebellar levels are indeed higher in C9ORF72 expansion carriers than in (disease) controls (table 2, figure 1). In addition, in the frontal cortex, we showed that TTR levels are elevated in patients with a $C 9 O R F 72$ repeat expansion as compared to controls (table 2). Of note, we also performed a sensitivity analysis to assess whether our findings could have been biased by differences in age at death. It is important that similar findings were observed when restricting our analysis to a subset of participants with a comparable age at death (not shown). In addition, given the low levels of TTR and HOXA5, especially in (disease) controls, we also validated their upregulation in a subset of participants using other techniques, including digital molecular barcoding (not shown) and previously published RNA sequencing data (figure e-1). ${ }^{20}$

The vast amount of C9ORF72 expression data available for this cohort then allowed us to determine whether the levels of $C 9 O R F 72$ transcripts were associated with the levels of HOXA5 and TTR. In our overall cohort (expansion carriers, disease controls, and controls), lower cerebellar levels of C9ORF72 transcript variant 2 were associated with higher cerebellar levels of both HOXA5 $(r=-0.60, p=3.21 \mathrm{e}-$ 09 , Spearman test of correlation) and TTR $(r=$ $-0.47, p=2.21 \mathrm{e}-06$, Spearman test of correlation, table 3), which is not surprising given the fact that expansion carriers demonstrate decreased levels of C9ORF72 transcript variant $2 .{ }^{11}$ More excitingly, we noticed that higher cerebellar levels of introncontaining transcripts (both intron 1a and intron 1b) are associated with higher cerebellar levels of HOXA5 transcripts (intron 1a: $r=0.43, p=$ 6.47e-05, intron 1b: $r=0.36, p=0.0008$, Spearman test of correlation, table 3$)$. In the frontal cortex, lower C9ORF72 variant 2 levels were also associated with higher TTR levels $(r=-0.28, p=0.006$, Spearman test of correlation, table 3 ).

\section{Associations of C9ORF72 transcripts with HOXA5 and} $T T R$ transcripts in expansion carriers. Because we were able to validate our findings related to HOXA5 and $T T R$ and detect significant associations with specific C9ORF72 transcripts in our overall cohort, we then evaluated the presence of any potential associations within our cohort of C9ORF72 expansion carriers. In the cerebellum, increased levels of total C9ORF72 transcripts were associated with increased HOXA5 transcripts $(r=0.51, p=8.86 \mathrm{e}-05$, Spearman test of correlation, table 4), most prominently in patients with a pathologic diagnosis of FTLD $(r=0.65$, 
Table 3 Associations of HOXA5 and TTR transcripts with C9ORF72 transcripts in the overall cohort

\begin{tabular}{|c|c|c|c|c|}
\hline \multirow[b]{2}{*}{ TaqMan } & \multirow[b]{2}{*}{ Group } & \multirow[b]{2}{*}{ Association } & \multicolumn{2}{|l|}{ Overall } \\
\hline & & & Spearman $r(95 \% \mathrm{Cl})$ & $p$ Value \\
\hline \multirow[t]{12}{*}{ Cerebellum } & HOXA5 & Total & $-0.21(-0.40$ to 0.02$)$ & 0.07 \\
\hline & & Variant 1 & $-0.19(-0.39$ to 0.03$)$ & 0.09 \\
\hline & & Variant 2 & $-0.60(-0.73$ to -0.42$)$ & $3.21 \mathrm{e}-09$ \\
\hline & & Variant 3 & $0.06(-0.14$ to 0.27$)$ & 0.56 \\
\hline & & Intron 1a & $0.43(0.23$ to 0.60$)$ & $6.47 e-05$ \\
\hline & & Intron $1 \mathrm{~b}$ & 0.36 (0.16 to 0.54$)$ & 0.0008 \\
\hline & TTR & Total & $-0.19(-0.39$ to 0.02$)$ & 0.06 \\
\hline & & Variant 1 & $-0.20(-0.40$ to 0.003$)$ & 0.05 \\
\hline & & Variant 2 & $-0.47(-0.62$ to -0.28$)$ & $2.21 \mathrm{e}-06$ \\
\hline & & Variant 3 & $-0.03(-0.24$ to 0.18$)$ & 0.78 \\
\hline & & Intron $1 \mathrm{a}$ & 0.23 (0.03 to 0.42$)$ & 0.03 \\
\hline & & Intron $1 \mathrm{~b}$ & $0.23(0.04$ to 0.41$)$ & 0.03 \\
\hline \multirow[t]{6}{*}{ Frontal cortex } & TTR & Total & $-0.15(-0.35$ to 0.07$)$ & 0.16 \\
\hline & & Variant 1 & $-0.06(-0.25$ to 0.14$)$ & 0.58 \\
\hline & & Variant 2 & $-0.28(-0.47$ to -0.08$)$ & 0.006 \\
\hline & & Variant 3 & $-0.17(-0.35$ to 0.03$)$ & 0.09 \\
\hline & & Intron 1a & 0.23 (0.02 to 0.42 ) & 0.03 \\
\hline & & Intron 1b & $0.14(-0.07$ to 0.34$)$ & 0.19 \\
\hline
\end{tabular}

Data are Spearman correlation coefficient $r$ (95\% confidence interval [Cl]) or $p$ value; HOXA5 and TTR transcript levels are normalized to the geometric mean of endogenous control genes ribosomal protein, large, PO (RPLPO), and glyceraldehyde-3-phosphate dehydrogenase (GAPDH). In total, in our overall cohort (expansion carriers, disease controls, and controls), we examined 6 different associations (total C9ORF72 transcripts, C9ORF72 transcript variant 1, C9ORF72 transcript variant 2, C9ORF72 transcript variant 3 , intron 1a-containing C9ORF72 transcripts, and intron 1b-containing C9ORF72 transcripts) for each outcome, and thus, $p$ values below 0.0083 were considered significant after Bonferroni correction. A Spearman test of correlation was used $(p<0.0083$ considered significant after Bonferroni correction). Similar findings were obtained when normalizing to neuronal markers (not shown for simplicity).

$p=0.0009$, Spearman test of correlation). Of interest, we also noticed that elevated levels of introncontaining transcripts were associated with elevated levels of HOXA5 or TTR (table 4). For HOXA5, this association (intron 1a: $r=0.60, p=1.61 \mathrm{e}-06$, intron $1 \mathrm{~b}: r=0.54, p=2.75 \mathrm{e}-05$, Spearman test of correlation, table 4) was driven by patients with a pathologic diagnosis of FTLD (intron 1a: $r=0.72$, $p=0.0001$, intron $1 \mathrm{~b}: r=0.59, p=0.003$, Spearman test of correlation). For TTR, however, the association (intron 1a: $r=0.40, p=0.003$, intron $1 \mathrm{~b}: r=0.43, p=0.001$, Spearman test of correlation, table 4) was most profound in patients with a pathologic diagnosis of MND (intron 1a: $r=0.83$, $p=0.0002$, intron $1 \mathrm{~b}: r=0.88, p=3.78 \mathrm{e}-05$, Spearman test of correlation).

Because we previously discovered associations between dipeptide repeat proteins and introncontaining transcripts, ${ }^{11}$ we subsequently evaluated potential associations with poly(GP) and poly(GA) proteins, which can form abundant inclusions in the neocortical regions, hippocampus, thalamus, and cerebellum. ${ }^{3,4,9,21-24}$ Although no significant associations were detected for TTR, we did observe an association for HOXA5: higher levels of dipeptide repeat proteins were associated with higher levels of HOXA5 (poly[GP]: $r=0.52, p=0.0002$, poly[GA]: $r=0.56, p=3.89 \mathrm{e}-05$, Spearman test of correlation, table 4).

In the frontal cortex, we noted a trend between C9ORF72 variant 2 and TTR $(r=-0.39, p=$ 0.004 , Spearman test of correlation), particularly in the subset of patients with a pathologic diagnosis of FTLD $(r=-0.62, p=0.002$, Spearman test of correlation, table 4).

In both brain regions, we did not detect significant associations with other variables, such as expansion size (table 4), disease subgroup, sex, age at onset, age at death, or survival after onset (not shown).

\section{Cerebellar changes in TTR transcripts are reflected by} changes in protein levels. Given the fact that TTR is an extracellular protein, we determined whether changes in RNA levels were reflected by changes in protein levels, which could indicate that TTR may serve as a biomarker for C9ORF72-related diseases. Because our findings were most profound in the cerebellum, we extracted protein from this neuroanatomic region and performed Western blots. As expected, we detected a significant increase in cerebellar TTR protein levels in patients with a repeat expansion (mean $174 \% \pm 34 \%)$ as compared to patients without a repeat expansion (mean $100 \% \pm 18 \%, p<0.05$, 2-sample $t$ test, figure 1). We also performed immunohistochemistry to examine whether an aggregated form of the TTR protein was present in the cerebellum because TTR protein aggregates have been reported in other diseases, such as familial amyloid polyneuropathy. ${ }^{25}$ We observed diffuse cytoplasmic TTR staining in pyramidal neurons and Purkinje cells, and in the neuropil; however, no TTR deposits were detected similar to those seen in patients with TTR amyloidosis (not shown).

To further evaluate TTR as a potential biomarker, we determined its protein levels in the CSF. In our first cohort, the median TTR protein level in expansion carriers was $15.5 \mu \mathrm{g} / \mathrm{mL}$ (interquartile range [IQR] 13.7-17.6) and in the remaining participants $16.3 \mu \mathrm{g} / \mathrm{mL}$ (IQR 14.5-17.7), which was not significantly different ( $p=0.29$, Wilcoxon rank-sum test). Our second cohort revealed a median TTR protein level of $12.5 \mu \mathrm{g} / \mathrm{mL}$ in expansion carriers (IQR 11.012.6) and $12.3 \mu \mathrm{g} / \mathrm{mL}$ in other participants (IQR 11.8-14.4); again, this difference did not reach statistical significance $(p=0.58$, Wilcoxon rank-sum 
Table 4 Associations of HOXA5 and TTR transcripts with C9ORF72 transcripts, expansion size, and dipeptide repeat proteins in expansion carriers

\begin{tabular}{|c|c|c|c|c|c|c|c|c|c|c|}
\hline \multirow[b]{2}{*}{ TaqMan } & \multirow[b]{2}{*}{ Group } & \multirow[b]{2}{*}{ Association } & \multicolumn{2}{|l|}{ C9Plus cohort } & \multicolumn{2}{|l|}{ FTLD cohort } & \multicolumn{2}{|l|}{ FTLD/MND cohort } & \multicolumn{2}{|l|}{ MND cohort } \\
\hline & & & Spearman r $(95 \% \mathrm{Cl})$ & $p$ Value & Spearman $r(95 \% \mathrm{Cl})$ & $p$ Value & Spearman r $(95 \% \mathrm{Cl})$ & $p$ Value & Spearman r (95\% Cl) & $p$ Value \\
\hline \multirow[t]{18}{*}{ Cerebellum } & HOXA5 & Total & 0.51 (0.29 to 0.68$)$ & $8.86 e-05$ & 0.65 (0.32 to 0.83 ) & 0.0009 & $0.20(-0.40$ to 0.65$)$ & 0.47 & $0.43(-0.19$ to 0.86$)$ & 0.13 \\
\hline & & Variant 1 & 0.33 (0.08 to 0.54$)$ & 0.01 & 0.50 (0.11 to 0.78 ) & 0.01 & $-0.12(-0.68$ to 0.44$)$ & 0.67 & $0.16(-0.45$ to 0.68$)$ & 0.59 \\
\hline & & Variant 2 & $-0.13(-0.40$ to 0.15$)$ & 0.33 & $-0.07(-0.51$ to 0.41$)$ & 0.77 & $-0.19(-0.68$ to 0.36$)$ & 0.50 & $-0.08(-0.67$ to 0.50$)$ & 0.80 \\
\hline & & Variant 3 & 0.38 (0.13 to 0.59 ) & 0.005 & $0.35(-0.12$ to 0.69$)$ & 0.10 & $0.41(-0.16$ to 0.81$)$ & 0.12 & $0.22(-0.42$ to 0.77$)$ & 0.44 \\
\hline & & Intron 1a & 0.60 (0.37 to 0.77$)$ & $1.61 \mathrm{e}-06$ & 0.72 (0.39 to 0.89 ) & 0.0001 & $0.56(-0.02$ to 0.91$)$ & 0.03 & $0.41(-0.21$ to 0.82$)$ & 0.14 \\
\hline & & Intron 1b & 0.54 (0.31 to 0.71$)$ & $2.75 e-05$ & 0.59 (0.20 to 0.82$)$ & 0.003 & 0.65 (0.17 to 0.88$)$ & 0.009 & $0.42(-0.13$ to 0.80$)$ & 0.14 \\
\hline & & C9ORF72 expansion size & $-0.17(-0.42$ to 0.10$)$ & 0.24 & $-0.09(-0.53$ to 0.36$)$ & 0.68 & $-0.42(-0.70$ to 0.02$)$ & 0.12 & $-0.18(-0.64$ to 0.44$)$ & 0.55 \\
\hline & & Poly(GP) & 0.52 (0.26 to 0.72$)$ & 0.0002 & $0.32(-0.12$ to 0.71$)$ & 0.15 & $0.50(-0.15$ to 0.91$)$ & 0.08 & $0.45(-0.09$ to 0.84$)$ & 0.10 \\
\hline & & Poly(GA) & $0.56(0.33-0.73)$ & $3.89 e-05$ & 0.60 (0.20 to 0.85$)$ & 0.004 & $0.41(-0.26$ to 0.84$)$ & 0.17 & $0.09(-0.59$ to 0.62$)$ & 0.74 \\
\hline & TTR & Total & $0.25(-0.02$ to 0.50$)$ & 0.07 & 0.44 (0.08 to 0.70$)$ & 0.03 & $-0.02(-0.62$ to 0.60$)$ & 0.95 & $0.35(-0.23$ to 0.83$)$ & 0.22 \\
\hline & & Variant 1 & $0.07(-0.23$ to 0.36$)$ & 0.60 & $0.29(-0.13$ to 0.62$)$ & 0.18 & $-0.18(-0.74$ to 0.47$)$ & 0.53 & $0.03(-0.52$ to 0.61$)$ & 0.91 \\
\hline & & Variant 2 & $-0.17(-0.43$ to 0.12$)$ & 0.21 & $-0.06(-0.53$ to 0.42$)$ & 0.77 & $-0.24(-0.74$ to 0.36$)$ & 0.40 & $-0.37(-0.78$ to 0.21$)$ & 0.19 \\
\hline & & Variant 3 & 0.11 (-0.19 to 0.39$)$ & 0.43 & 0.10 (-0.34 to 0.52$)$ & 0.65 & $0.05(-0.61$ to 0.67$)$ & 0.85 & $0.24(-0.36$ to 0.75$)$ & 0.41 \\
\hline & & Intron 1a & 0.40 (0.15 to 0.59$)$ & 0.003 & $0.27(-0.16$ to 0.66$)$ & 0.22 & $0.28(-0.31$ to 0.73$)$ & 0.31 & 0.83 (0.54 to 0.96 ) & 0.0002 \\
\hline & & Intron $1 b$ & 0.43 (0.18 to 0.63 ) & 0.001 & $0.37(-0.04$ to 0.67$)$ & 0.08 & $0.33(-0.32$ to 0.80$)$ & 0.23 & 0.88 (0.62 to 0.97 ) & $3.78 \mathrm{e}-05$ \\
\hline & & C9ORF72 expansion size & $0.01(-0.29$ to 0.30$)$ & 0.93 & $0.05(-0.38$ to 0.45$)$ & 0.83 & $0.12(-0.57$ to 0.66$)$ & 0.67 & $0.01(-0.66$ to 0.64$)$ & 0.96 \\
\hline & & Poly(GP) & $0.09(-0.19$ to 0.36$)$ & 0.55 & $-0.11(-0.49$ to 0.30$)$ & 0.62 & $0.19(-0.43$ to 0.66$)$ & 0.54 & $0.38(-0.15$ to 0.76$)$ & 0.17 \\
\hline & & Poly(GA) & $-0.02(-0.29$ to 0.26$)$ & 0.91 & $0.15(-0.26$ to 0.54$)$ & 0.50 & $0.00(-0.60$ to 0.63$)$ & 0.99 & $-0.002(-0.53$ to 0.57$)$ & 1.00 \\
\hline \multirow[t]{8}{*}{ Frontal cortex } & TTR & Total & $-0.19(-0.47$ to 0.11$)$ & 0.17 & $-0.05(-0.51$ to 0.43$)$ & 0.84 & $-0.33(-0.79$ to 0.24$)$ & 0.26 & $-0.39(-0.86$ to 0.30$)$ & 0.19 \\
\hline & & Variant 1 & $-0.03(-0.32$ to 0.26$)$ & 0.82 & $-0.09(-0.40$ to 0.56$)$ & 0.69 & $-0.12(-0.58$ to 0.40$)$ & 0.70 & 0.01 (-0.61 to 0.60$)$ & 0.96 \\
\hline & & Variant 2 & $-0.39(-0.59$ to -0.14$)$ & 0.004 & $-0.62(-0.80$ to -0.31$)$ & 0.002 & $-0.51(-0.90$ to 0.07$)$ & 0.06 & -0.11 (-0.68 to 0.53$)$ & 0.73 \\
\hline & & Variant 3 & $-0.03(-0.31$ to 0.26$)$ & 0.81 & $0.32(-0.10$ to 0.67$)$ & 0.14 & $-0.33(-0.79$ to 0.30$)$ & 0.26 & 0.08 (-0.61 to 0.68$)$ & 0.80 \\
\hline & & Intron 1a & $0.18(-0.08$ to 0.41$)$ & 0.21 & $0.31(-0.14$ to 0.70$)$ & 0.16 & $0.14(-0.39$ to 0.58$)$ & 0.63 & $0.21(-0.46$ to 0.75$)$ & 0.49 \\
\hline & & Intron $1 b$ & $-0.02(-0.31$ to 0.30$)$ & 0.88 & $-0.08(-0.52$ to 0.47$)$ & 0.72 & $-0.08(-0.71$ to 0.51$)$ & 0.79 & $0.22(-0.54$ to 0.80$)$ & 0.47 \\
\hline & & C9ORF72 expansion size & $0.02(-0.28$ to 0.30$)$ & 0.91 & $-0.25(-0.62$ to 0.19$)$ & 0.26 & $0.49(-0.08$ to 0.83$)$ & 0.07 & $-0.19(-0.80$ to 0.48$)$ & 0.55 \\
\hline & & Poly(GP) & $0.15(-0.15$ to 0.42$)$ & 0.32 & $0.40(-0.10$ to 0.71$)$ & 0.08 & $0.43(-0.14$ to 0.88$)$ & 0.12 & $0.05(-0.63$ to 0.65$)$ & 0.85 \\
\hline
\end{tabular}

Abbreviations: FTLD = frontotemporal lobar degeneration; MND $=$ motor neuron disease

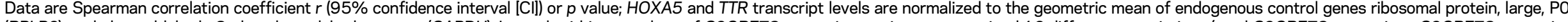

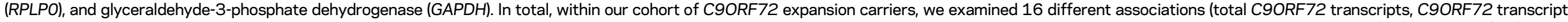

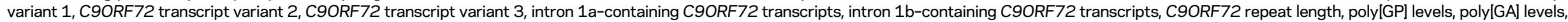

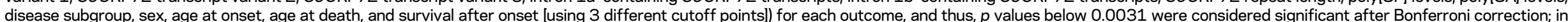

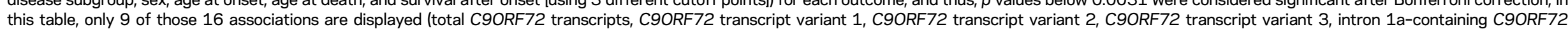

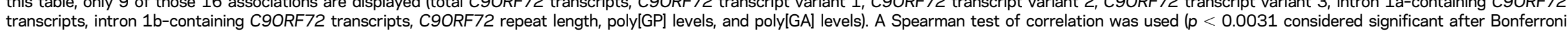
transcripts, intron $1 \mathrm{~b}$-containing C9ORF72 transcripts, C9ORF72 repeat length, poly[GP] levels, and poly[GA]
correction). Similar findings were obtained when normalizing to neuronal markers (not shown for simplicity). 
test). Of note, similar findings were obtained when adjusting for possible confounders, when removing outliers, and when restricting our analysis to specific (sub)groups (e.g., symptomatic participants).

Loss of C9ORF72 expression increases HOXA5 and TTR transcripts. Next, we performed cell culture experiments to determine which C9ORF72-related disease characteristics might drive the specific upregulation of HOXA5 and TTR. Of interest, we observed an increase of $H O X A 5$ in response to a knock-down of total C9ORF72 transcripts (mean 193\% $\pm 3 \%, p=$ 0.0002 , 2-sample $t$ test). A comparable effect was seen on $T T R$ (mean $129 \% \pm 6 \%, p=0.01,2$ sample $t$ test) after knocking down total C9ORF72 transcripts. Given our significant findings related to C9ORF72 transcript variant 2, we then targeted variant 2. It is important that knocking down of variant 2 was sufficient to increase HOXA5 levels (mean 274\% $\pm 12 \%, p=0.005$, 2-sample $t$ test). For TTR, targeting C9ORF72 variant 2 also affected $T T R$ levels (mean 293\% $\pm 37 \%, p=0.03,2$ sample $t$ test). Overexpression of full-length C9ORF72 or expression of 66 GGGGCC repeats, however, did not seem to affect HOXA5 or TTR (not shown). Consequently, it seems that a loss of C9ORF72 expression triggers an increase in HOXA5 and TTR.

DISCUSSION We set out to increase our understanding of C9ORF72-related diseases and revealed elevated levels of multiple homeobox genes (e.g., HOXA5) and TTR. Of interest, our findings were most profound in the cerebellum, a region without substantial neuronal loss that demonstrates pathologic characteristics of diseases linked to a repeat expansion in C9ORF72., ${ }^{3,8}$ In fact, in this neuroanatomic region, associations with neuropathologic and clinical features of the disease have already been reported. It has been shown, for instance, that dipeptide repeat protein levels are lower in pathologically diagnosed patients with MND as compared to patients with FTLD, ${ }^{9,10}$ and that they are associated with the cognitive score of clinically diagnosed patients with ALS. ${ }^{10}$ Moreover, an association between dipeptide repeat proteins and introncontaining transcripts has been described in the cerebellum, indicating that transcripts containing the entire first intron may serve as templates for repeat-associated non-ATG translation. ${ }^{11}$ In addition, extensive alternative splicing and polyadenylation defects have been reported in the cerebellum of C9ORF72 expansion carriers. ${ }^{20}$ The fact that our findings were most prominent in the cerebellum emphasizes that this region may have been underappreciated and could play an important role in C9ORF72-related diseases.
The upregulation of multiple homeobox genes and TTR was further substantiated by the results of our gene ontology analysis that revealed enrichment for developmental processes. The observed enrichment for developmental processes is not surprising given the function of homeobox genes and TTR in neuronal development and neuroprotection. ${ }^{14-19}$ Homeobox genes, for example, are involved in neuronal specification and target connectivity; they play a key role in the identity, organization, and peripheral connectivity of motor neuron subtypes. ${ }^{14}$ TTR is important for the transportation of thyroid hormones (thyroxine [T4]) and retinol (vitamin A), and in addition, it is thought to participate in behavior, maintenance of normal cognitive processes during aging, neuropeptide processing, and nerve regeneration. ${ }^{26}$ Of interest, homeobox genes and TTR are linked. For instance, an active metabolite of vitamin A (alltrans retinoic acid) functions in maintenance of many processes (e.g., brain function) and is essential for limb and organ development through homeobox gene-mediated mechanisms. ${ }^{27}$

Although the relative differences we observed in HOXA5 and TTR are a reflection of small absolute differences, the fact that their levels are barely detectable in (disease) controls raises the possibility that their expression may have been (re)activated in the adult brain. Such a (re)activation could be specific to C9ORF72-related diseases and might be driven by certain mechanisms underlying those diseases. It is currently unknown where the elevated HOXA5 and TTR levels are coming from; a change in celltype composition, invading progenitor cells, and cell-autonomous effects need to be taken into consideration.

One of the pathologic hallmarks of C9ORF72related diseases is a reduction in C9ORF72 expression levels. ${ }^{1}$ Because the most prominent decrease has been reported for C9ORF72 variant 2 transcripts, ${ }^{11}$ one could speculate that levels of this transcript might be associated with levels of HOXA5 or TTR. Indeed, in our overall cohort, we demonstrated that lower levels of C9ORF72 variant 2 transcripts were associated with higher levels of HOXA5 and TTR transcripts. We also detected associations with intron-containing transcripts; in our expansion carriers, for instance, higher levels of intron-containing transcripts were associated with $H O X A 5$ transcript levels, especially in patients with a pathologic diagnosis of FTLD. In addition, HOXA5 transcript levels were associated with dipeptide repeat proteins, which is in agreement with reports that describe associations between intron-containing transcripts and dipeptide repeat proteins. ${ }^{11}$ For TTR, we also observed a correlation with intron-containing transcripts, but this association was most profound in patients with 
a pathologic diagnosis of MND; no significant associations were observed with dipeptide repeat proteins. Thus, although some associations were shared between HOXA5 and TTR, others differed.

We emphasize that in our study, as in any observational study, performing association analysis between 2 variables is not intended to provide information about possible mechanisms, but rather is intended to address the initial question whether 2 variables are related in any way. It is only after this initial question is addressed that further questions, such as mechanism, become relevant. To determine what may have driven an increase in HOXA5 and TTR, we already performed cell culture experiments, demonstrating that lower levels of C9ORF72 resulted in higher levels of HOXA5 and TTR. These effects were observed when targeting either total C9ORF72 transcripts or C9ORF72 transcript variant 2; we cannot, however, exclude nonspecific effects on other transcript variants, and further studies are warranted. Additional studies could also help to learn more about mechanisms that link C9ORF72 to HOXA5 and TTR, particularly because little is known about the function, and interaction partners, of C9ORF72. Moreover, future studies could examine downstream targets and/or upstream regulators that might contribute to the observed differences.

It is important that TTR protein levels have been evaluated as a potential biomarker for ALS and FTD, ${ }^{28-33}$ but findings were inconsistent, which could, in part, be explained by the genetic, pathologic, and clinical heterogeneity observed in those patients. Although our results seem to indicate that cerebellar TTR protein levels are elevated in C9ORF72 expansion carriers, we could not detect significant differences in CSF TTR protein levels. The lack of a significant difference could be due to the presence of posttranslational modifications to the TTR protein that are undetectable using our immunoassay. Alternatively, it might be possible that the secretory pathway is affected, hampering the secretion of TTR into the CSF. Future experiments using mass spectrometry and immunoassays with different antibodies as well as experiments investigating the secretion of TTR (e.g., in cell culture models) should be used to test these hypotheses. In addition, future studies should examine whether TTR protein levels are associated with features of the disease (e.g., in the CSF or plasma) and whether they change over time, especially because one could postulate that a single time point in a clinical cohort may not reflect changes observed in a pathologic cohort (end-stage disease).

Thus, we discovered elevated levels of multiple homeobox genes and TTR, reported to be involved in developmental processes and neuroprotection, in brain tissue obtained from C9ORF72 expansion carriers. Our findings may point to the presence of compensatory mechanisms aiming to mitigate the progression of C9ORF72-related diseases.

\section{AUTHOR CONTRIBUTIONS}

NiCole A. Finch, Matthew C. Baker, Tania F. Gendron, Kevin F. Bieniek, Mariely DeJesus-Hernandez, Patricia H. Brown, Jeannie Chew, Karen R. Jansen-West, Lillian M. Daughrity, Alexandra M. Nicholson, Melissa E. Murray, and Robert Bowser: acquisition of data, analysis or interpretation of data, and revising the manuscript for content, including writing of content. Xue Wang and Yan W. Asmann: analysis or interpretation of data and drafting the manuscript for content, including writing of content. Michael G. Heckman: analysis or interpretation of data, statistical analysis, and drafting the manuscript for content, including writing of content. Joanne Wuu: contribution of vital reagents/tools/patients and revising the manuscript for content, including writing of content. Keith A. Josephs, Joseph E. Parisi, David S. Knopman, Ronald C. Petersen, Leonard Petrucelli, Bradley F. Boeve, Neill R. Graff-Radford, Dennis W. Dickson, Michael Benatar, and Kevin B. Boylan: revising the manuscript for content, including writing of content, contribution of vital reagents/tools/patients, and obtaining funding. Rosa Rademakers: study concept or design, acquisition of data, analysis or interpretation of data, drafting the manuscript for content, including writing of content, revising the manuscript for content, including writing of content, study supervision or coordination, and obtaining funding. Marka van Blitterswijk: study concept or design, acquisition of data, analysis or interpretation of data, statistical analysis, drafting the manuscript for content, including writing of content, revising the manuscript for content, study supervision or coordination, and obtaining funding.

\section{ACKNOWLEDGMENT}

The authors thank Dr. J. Jiang, Dr. C. Lagier-Tourenne, Dr. D. Edbauer, and Dr. D.W. Cleveland for providing antibodies used to measure dipeptide repeat proteins. In addition, they thank Dr. M. Prudencio and Dr. V. V. Belzil for sharing RNA sequencing results.

\section{STUDY FUNDING}

Supported by the National Institutes of Health (R21 NS093118, R01 NS080882, R35 NS097261, R35 NS097273, P50 AG016574, P01 NS084974, and R56 NS061867) and the ALS Therapy Alliance. Dr. van Blitterswijk is a former recipient of the Milton Safenowitz Post-Doctoral Fellowship for ALS Research from the ALS Association and is currently supported by the Clinical Research in ALS and Related Disorders for Therapeutic Development (CReATe) Clinical Research Fellowship. CReATe (U54 NS092091) is part of the Rare Diseases Clinical Research Network (RDCRN), an initiative of the Office of Rare Diseases Research (ORDR) and National Center for Advancing Translational Sciences (NCATS). CReATe is funded through collaboration between NCATS and the National Institute of Neurological Disorders and Stroke.

\section{DISCLOSURE}

NiCole A. Finch and Xue Wang report no disclosures. Matthew C. Baker holds the following patents: US Patent No. 12/302,691 (Detecting and Treating Dementia [2008]) and US Patent No. 12/413,869 (Methods and Materials for Detecting and Treating Dementia [2009]). Michael G. Heckman has served on the editorial board of Parkinsonism \& Related Disorders. Tania F. Gendron has received speaker honoraria from Johns Hopkins; holds a patent for Methods and materials for detecting poly (GP) proteins in tissues from C9ORF72 repeat expansion carriers; has received research support from NIH, the ALS Association, and the Muscular Dystrophy Association; and receives license fee payments for C9ORF72 repeat expansion constructs and viruses, and for antibodies against C9ORF72 dipeptide repeat proteins. Kevin F. Bieniek reports no disclosures. Joanne Wuu has received research support from the Muscular Dystrophy Association, the NIH, the Food and Drug Administration, Eli Lilly and Company, the University of Miami, Department of Neurology, the ALS Association, and the Department of Defense. Mariely DeJesusHernandez holds a patent on Methods to screen for the hexanucleotide repeat expansion in the C9ORF72 gene. Patricia H. Brown, Jeannie 
Chew, Karen R. Jansen-West, and Lillian M. Daughrity report no disclosures. Alexandra M. Nicholson has received research support from the Association for Frontotemporal Degeneration. Melissa E. Murray has served on the editorial boards of BMC Neurology and Frontiers in Neurology and has been a consultant for Avid Radiopharmaceuticals. Keith A. Josephs receives research support from the NIH, the Dana Foundation, and the Alzheimer's Association; and is an editorial board member for Acta Neuropathologica and Journal of Neuropathology \& Experimental Neurology. Joseph E. Parisi has served on the Defense Health Board Health Care Delivery Subcommittee; has received publishing royalties from Oxford University Press; and has received research support from the NIH. David S. Knopman has served on the scientific advisory boards of the Bluefield Project, Lundbeck Pharmaceuticals, and DIAN study DSMB; served on a Data Safety Monitoring Board for Lundbeck Pharmaceuticals and the DIAN study; was an investigator in clinical trials sponsored by Lilly, TauRx, and Biogen Pharmaceuticals; has received travel funding/speaker honoraria from the Alzheimer Conference (Seoul, Korea) and the Behavioral Neurology Conference (Hyderabad, India); has served on the editorial board of Neurology; and has received research support from the NIH. Ronald C. Petersen has served on the scientific advisory boards of Pfizer, Janssen Alzheimer Immunotherapy, Elan Pharmaceuticals, Wyeth Pharmaceuticals, and GE Healthcare; has received publishing royalties from Oxford University Press; has been a consultant for Roche Incorporated, Merck, GeneTech, Biogen, and Eli Lilly; has received research support from the NIH; and has served on the National Advisory Council on Aging. Leonard Petrucelli has received research support from the Mayo Clinic Foundation, the NIH, the ALS Association, Lundbeck, Biogen, Robert Packard Center for ALS Research at Johns Hopkins, Target ALS, the Association for Frontotemporal Degeneration, and the Department of Defense; has received license fee payments from Lundbeck, Biogen, and Denali; has received royalty payments for the licensing of TDP-43 antibody; has served on the scientific advisory boards of SAB, Denali SAB, and Biogen; and serves on the editorial boards of the Journal of Neuroscience, Molecular Neurodegeneration, and PLoS One. Bradley F. Boeve has served as an investigator for clinical trials sponsored by GE Healthcare and FORUM Pharmaceuticals; receives royalties from the publication of a book entitled Behavioral Neu rology of Dementia (Cambridge Medicine, 2009); serves on the Scientific Advisory Board of the Tau Consortium; has consulted for Isis Pharmaceuticals and Ionis Pharmaceuticals; and receives research support from the NIH, GE Healthcare, FORUM Pharmaceuticals, C2N Diagnostics, the Little Family Foundation, and the Mangurian Foundation. Neill R. Graff-Radford has served on the editorial board of Alzheimer's Research \& Therapy; has received publishing royalties from $U_{p} T o D a t e$; has consulted for Cytox; and has received research support from TauRx, Lilly, Biogen, Axovant, and the NIH. Yan W. Asmann reports no disclosures relevant to this manuscript. Dennis W. Dickson has served on the editorial boards of Acta Neuropathologica, Brain, Brain Pathology, Neurobiology of Aging, Annals of Neurology, Neuropathology, International Journal of Clinical and Experimental Pathology, and American Journal of Neurodegenerative Disease; has received travel funding and speaker honoraria from Novartis; and has received research support from the NIH, the Society for PSP: Foundation for PSP/CBD and Related Disorders, and the Mangurian Foundation. Michael Benatar has served on the scientific advisory boards of Denali, Ra Pharmaceuticals, Alnylam, and Mitsubishi Tanabe; has served on the editorial board of Journal Watch Neurology; has been consultant for Congressionally Directed Medical Research Program (CDMRP) ALS Research Program Integration Panel; has received research funding from the Muscular Dystrophy Association, the ALS Association, the NIH, the Food and Drug Administration, the Department of Defense, Cytokinetics Inc., Alexion Pharmaceuticals, Kimmelman Estate, Eli Lilly and Company, Neuraltus, and the ALS Recovery Fund; and has had involvement in legal proceedings regarding Morris James LLP. Robert Bowser is a founder of Iron Horse Diagnostics, Inc. (a company focused on biomarkers related to ALS that holds patents on TTR as a biomarker for motor neuron diseases); has served on the scientific advisory boards of Denali Therapeutics and Above \& Beyond, LLC; has served on the editorial boards of Scientific Reports, the International Journal of Proteomics, and the American Journal of Neurodegenerative Disease; holds patents for Biomarkers for the diagnosis and prognosis of ALS, Biomarkers to monitor drug treatment of ALS and other neuromuscular disease patients, and Biomarkers for detecting and treating joint related pain; has been a consultant for Cytonics, Inc. and Merck; has received research support from the NIH, ALS Association, and Target ALS; and holds stock/stock options for Iron Horse Diagnostics, Inc. Kevin B. Boylan receives research support from the NIH, ALS Association, Genentech, Cytokinetics Inc., the Mayo Foundation, Neuraltus Pharmaceuticals, GlaxoSmithKline, Avanir Pharmaceuticals, and Synapse Biomedical. Rosa Rademakers receives research support from the NIH, the ALS Therapy Alliance, the Consortium for Frontotemporal Degeneration Research, the Mayo Clinic Udall Center of Excellence, and the Florida State Alzheimer's Disease Research grant; received honoraria for lectures or educational activities not funded by industry; serves on the medical advisory board of the Association for Frontotemporal Degeneration and on the board of directors of the International Society for Frontotemporal Dementia; and holds patents on Methods to screen for the hexanucleotide repeat expansion in the C9ORF72 gene, and Detecting and treating dementia. Marka van Blitterswijk receives research support from the NIH; is supported by the Clinical Research in ALS and Related Disorders for Therapeutic Development (CReATe) Clinical Research Fellowship; and is a former recipient of the Milton Safenowitz Post-Doctoral Fellowship for ALS research from the ALS Association. Go to Neurology.org/ng for full disclosure forms.

Received December 28, 2016. Accepted in final form April 18, 2017.

\section{REFERENCES}

1. DeJesus-Hernandez M, Mackenzie IR, Boeve BF, et al. Expanded GGGGCC hexanucleotide repeat in noncoding region of C9ORF72 causes chromosome 9p-linked FTD and ALS. Neuron 2011;72:245-256.

2. Renton AE, Majounie E, Waite A, et al. A hexanucleotide repeat expansion in C9ORF72 is the cause of chromosome 9p21-linked ALS-FTD. Neuron 2011;72:257-268.

3. Ash PE, Bieniek KF, Gendron TF, et al. Unconventional translation of C9ORF72 GGGGCC expansion Generates Insoluble Polypeptides specific to c9FTD/ALS. Neuron 2013;77:639-646.

4. Mori K, Weng SM, Arzberger T, et al. The C9orf72 GGGGCC repeat is translated into aggregating dipeptiderepeat proteins in FTLD/ALS. Science 2013;339:1335-1338.

5. Zhang K, Donnelly CJ, Haeusler AR, et al. The C9orf72 repeat expansion disrupts nucleocytoplasmic transport. Nature 2015;525:56-61.

6. Jovicic A, Mertens J, Boeynaems S, et al. Modifiers of C9orf72 dipeptide repeat toxicity connect nucleocytoplasmic transport defects to FTD/ALS. Nat Neurosci 2015; 18:1226-1229.

7. Freibaum BD, Lu Y, Lopez-Gonzalez R, et al. GGGGCC repeat expansion in C9orf72 compromises nucleocytoplasmic transport. Nature 2015;525:129-133.

8. Mizielinska S, Lashley T, Norona FE, et al. C9orf72 frontotemporal lobar degeneration is characterised by frequent neuronal sense and antisense RNA foci. Acta Neuropathol 2013;126:845-857.

9. Schludi MH, May S, Grasser FA, et al. Distribution of dipeptide repeat proteins in cellular models and C9orf72 mutation cases suggests link to transcriptional silencing. Acta Neuropathol 2015;130:537-555.

10. Gendron TF, van Blitterswijk M, Bieniek KF, et al. Cerebellar c9RAN proteins associate with clinical and neuropathological characteristics of C9ORF72 repeat expansion carriers. Acta Neuropathol 2015;130:559-573.

11. van Blitterswijk M, Gendron TF, Baker MC, et al. Novel clinical associations with specific C9ORF72 transcripts in patients with repeat expansions in C9ORF72. Acta Neuropathol 2015;130:863-876. 
12. van Blitterswijk M, Dejesus-Hernandez M, Niemantsverdriet $\mathrm{E}$, et al. Association between repeat sizes and clinical and pathological characteristics in carriers of C9ORF72 repeat expansions (Xpansize-72): a cross-sectional cohort study. Lancet Neurol 2013;12:978-988.

13. Gendron TF, Bieniek KF, Zhang YJ, et al. Antisense transcripts of the expanded C9ORF72 hexanucleotide repeat form nuclear RNA foci and undergo repeat-associated non-ATG translation in c9FTD/ALS. Acta Neuropathol 2013;126:829-844.

14. Philippidou P, Dasen JS. Hox genes: choreographers in neural development, architects of circuit organization. Neuron 2013;80:12-34.

15. Santos SD, Lambertsen KL, Clausen BH, et al. CSF transthyretin neuroprotection in a mouse model of brain ischemia. J Neurochem 2010;115:1434-1444.

16. Li X, Masliah E, Reixach N, Buxbaum JN. Neuronal production of transthyretin in human and murine Alzheimer's disease: is it protective? J Neurosci 2011;31:12483-12490.

17. Li X, Buxbaum JN. Transthyretin and the brain re-visited: is neuronal synthesis of transthyretin protective in $\mathrm{Alz}$ heimer's disease? Mol Neurodegener 2011;6:79.

18. Li X, Zhang X, Ladiwala AR, et al. Mechanisms of transthyretin inhibition of beta-amyloid aggregation in vitro. J Neurosci 2013;33:19423-19433.

19. Fleming CE, Saraiva MJ, Sousa MM. Transthyretin enhances nerve regeneration. J Neurochem 2007;103:831-839.

20. Prudencio M, Belzil VV, Batra R, et al. Distinct brain transcriptome profiles in C9orf72-associated and sporadic ALS. Nat Neurosci 2015;18:1175-1182.

21. Mori K, Arzberger T, Grasser FA, et al. Bidirectional transcripts of the expanded C9orf72 hexanucleotide repeat are translated into aggregating dipeptide repeat proteins. Acta Neuropathol 2013;126:881-893.

22. $\mathrm{Zu} \mathrm{T}$, Liu Y, Banez-Coronel M, et al. RAN proteins and RNA foci from antisense transcripts in C9ORF72 ALS and frontotemporal dementia. Proc Natl Acad Sci USA 2013;110:E4968-E4977.
23. Mann DM, Rollinson S, Robinson A, et al. Dipeptide repeat proteins are present in the $\mathrm{p} 62$ positive inclusions in patients with frontotemporal lobar degeneration and motor neurone disease associated with expansions in C9ORF72. Acta Neuropathol Commun 2013;1:68.

24. Mackenzie IR, Arzberger T, Kremmer E, et al. Dipeptide repeat protein pathology in C9ORF72 mutation cases: clinico-pathological correlations. Acta Neuropathol 2013; 126:859-879.

25. Plante-Bordeneuve V, Said G. Familial amyloid polyneuropathy. Lancet Neurol 2011;10:1086-1097.

26. Fleming CE, Nunes AF, Sousa MM. Transthyretin: more than meets the eye. Prog Neurobiol 2009;89:266-276.

27. Cunningham TJ, Duester G. Mechanisms of retinoic acid signalling and its roles in organ and limb development. Nat Rev Mol Cell Biol 2015;16:110-123.

28. Ryberg H, An J, Darko S, et al. Discovery and verification of amyotrophic lateral sclerosis biomarkers by proteomics. Muscle Nerve 2010;42:104-111.

29. Brettschneider J, Lehmensiek V, Mogel H, et al. Proteome analysis reveals candidate markers of disease progression in amyotrophic lateral sclerosis (ALS). Neurosci Lett 2010; 468:23-27.

30. Ranganathan S, Williams E, Ganchev P, et al. Proteomic profiling of cerebrospinal fluid identifies biomarkers for amyotrophic lateral sclerosis. J Neurochem 2005;95: 1461-1471.

31. Ruetschi U, Zetterberg H, Podust VN, et al. Identification of CSF biomarkers for frontotemporal dementia using SELDI-TOF. Exp Neurol 2005;196:273-281.

32. Hansson SF, Puchades M, Blennow K, Sjogren M, Davidsson P. Validation of a prefractionation method followed by two-dimensional electrophoresis—applied to cerebrospinal fluid proteins from frontotemporal dementia patients. Proteome Sci 2004;2:7.

33. Gloeckner SF, Meyne F, Wagner F, et al. Quantitative analysis of transthyretin, tau and amyloid-beta in patients with dementia. J Alzheimers Dis 2008;14:17-25. 


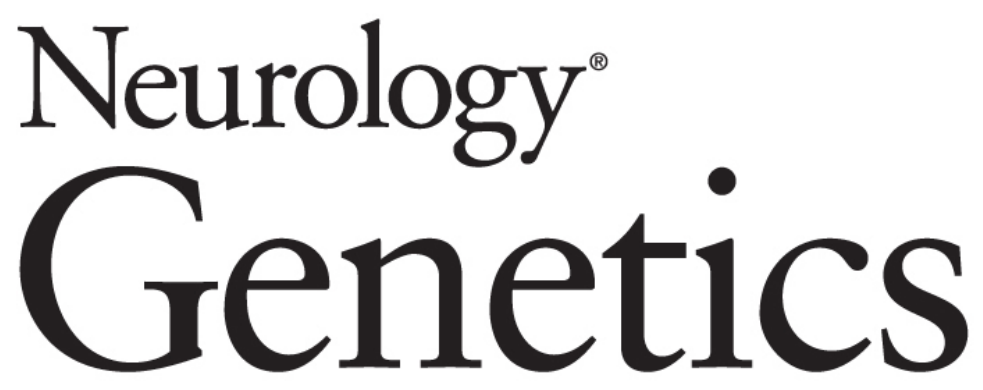

Abnormal expression of homeobox genes and transthyretin in C9ORF72 expansion carriers

NiCole A. Finch, Xue Wang, Matthew C. Baker, et al. Neurol Genet 2017;3;

DOI 10.1212/NXG.0000000000000161

This information is current as of June 7, 2017

Neurol Genet is an official journal of the American Academy of Neurology. Published since April 2015, it is an open-access, online-only, continuous publication journal. Copyright Copyright ( 2017 The Author(s). Published by Wolters Kluwer Health, Inc. on behalf of the American Academy of Neurology.. All rights reserved. Online ISSN: 2376-7839.

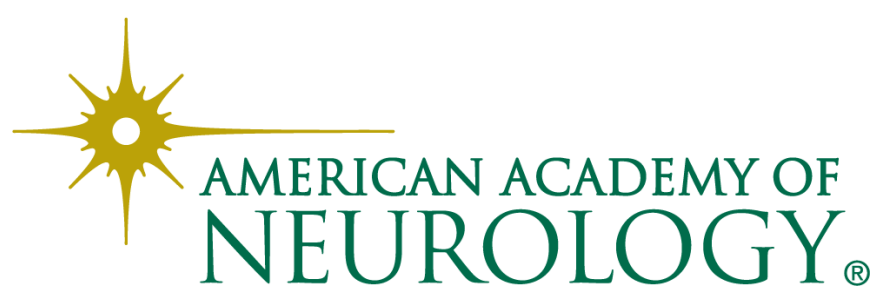




\section{Updated Information \& Services}

\section{Supplementary Material}

\section{References}

Citations

Subspecialty Collections

Permissions \& Licensing

Reprints including high resolution figures, can be found at: http://ng.neurology.org/content/3/4/e161.full.html

Supplementary material can be found at: http://ng.neurology.org/content/suppl/2017/06/07/3.4.e161.DC1

This article cites 33 articles, 4 of which you can access for free at: http://ng.neurology.org/content/3/4/e161.full.html\#\#ref-list-1

This article has been cited by 6 HighWire-hosted articles: http://ng.neurology.org/content/3/4/e161.full.html\#\#otherarticles

This article, along with others on similar topics, appears in the following collection(s):

\section{All Genetics}

http://ng.neurology.org//cgi/collection/all_genetics

Amyotrophic lateral sclerosis

http://ng.neurology.org//cgi/collection/amyotrophic_lateral_sclerosis_ Frontotemporal dementia

http://ng.neurology.org//cgi/collection/frontotemporal_dementia

Information about reproducing this article in parts (figures,tables) or in its entirety can be found online at:

http://ng.neurology.org/misc/about.xhtml\#permissions

Information about ordering reprints can be found online:

http://ng.neurology.org/misc/addir.xhtml\#reprintsus

Neurol Genet is an official journal of the American Academy of Neurology. Published since April 2015, it is an open-access, online-only, continuous publication journal. Copyright Copyright $\odot 2017$ The Author(s). Published by Wolters Kluwer Health, Inc. on behalf of the American Academy of Neurology.. All rights reserved. Online ISSN: 2376-7839.

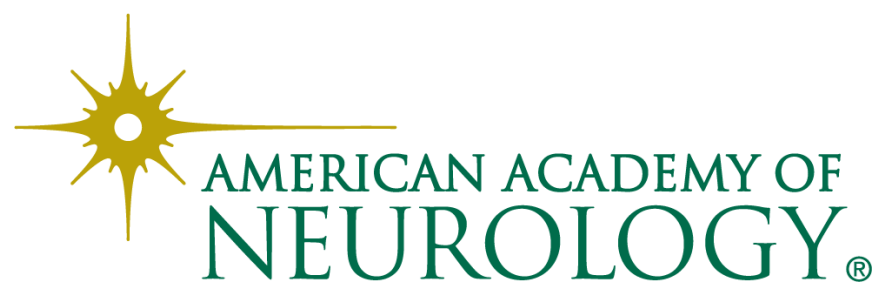

